\title{
Maître Pangloss
}

\section{Fréderic Deloffre}

\section{(2) OpenEdition \\ Journals}

\section{Édition électronique}

URL : https://journals.openedition.org/studifrancesi/40187

DOI : $10.4000 /$ studifrancesi. 40187

ISSN : 2427-5856

\section{Éditeur}

Rosenberg \& Sellier

\section{Édition imprimée}

Date de publication : 1 juillet 2004

Pagination : 133-140

ISSN : 0039-2944

\section{Référence électronique}

Fréderic Deloffre, "Maître Pangloss », Studi Francesi [En ligne], 142 (XLVIII | I) | 2004, mis en ligne le 30 novembre 2015, consulté le 09 septembre 2021. URL : http://journals.openedition.org/studifrancesi/ 40187 ; DOI : https://doi.org/10.4000/studifrancesi.40187

\section{(c) $(1)(9)$}

Studi Francesi è distribuita con Licenza Creative Commons Attribuzione - Non commerciale - Non opere derivate 4.0 Internazionale. 


\section{Maître Pangloss}

Lorsque nous avons proposé ${ }^{1}$ de rechercher le point de départ de la genèse de Candide dans l'expérience allemande de Voltaire, et spécialement dans sa rencontre avec Mme Bentinck, cette suggestion n'a éveillé, il faut l'avouer, que peu d'échos. Il est vrai qu'elle s'appuyait plutôt sur des intuitions et des vraisemblances que sur des analyses précises.

Certes, on avait signalé que le jeu des rencontres fortuites et des séparations entre Candide et Cunégonde reproduisait celui qui avait mis en présence et éloigné Voltaire et Mme Bentinck en 1740,1750-53 et 1758; on avait interprété la fuite de Candide, son engagement et sa désertion comme des allusions indéniables aux événements qui avaient amené Voltaire en Prusse et l'en avaient fait fuir; on avait rendu compte des lieux du roman, Thunder-ten-Tronch, Venise, Constantinople, par des transpositions, etc. Mais il restait à dégager le rôle de «matrice» des rencontres de Buckeburg, non seulement dans l'économie du roman, mais surtout dans la création par Voltaire des figures de deux de ses principaux personnages.

Nous voulons d'abord parler, bien entendu, de Mme Bentinck, qui avec les maîtres du château accueillit le visiteur lors de son premier séjour de 1740; ce point étant traité ailleurs ${ }^{2}$, nous n'y revenons pas. Il s'agit ici de Pangloss. Prétendre rendre compte de la création d'un tel personnage ne manque pas de hardiesse. Si Cunégonde joue un rôle important dans la structure du roman, celui qu'occupe Pangloss l'est en effet bien plus encore. N'est-il pas, sur le plan philosophique et théologique, l'incarnation même de la thèse que combat Voltaire? C'est lui qui donne vie à la doctrine de l'optimisme, en dépit ou à cause même du caractère absurde qu'elle revêt dans sa bouche. On est ici au cœur même du coup de génie d'où est sorti le roman. Il faut donc de puissants arguments pour fonder notre interprétation, selon laquelle le personnage de Pangloss aurait été inspiré à Voltaire par celui de Johann-Heinrich Meister, ou Jean-Henri Le Maître, prédicateur (Hofprediger), éducateur ou haut-précepteur (Erzieher, Hofmeister) d'une petite cour westphalienne, qu'il rencontra dans le château de Bückeburg, capitale du comté de Schaumburg-Lippe, à l'occasion de ses voyages en Prusse de 1740 et 1743.

La première condition à remplir pour valider la recherche est d'ordre matériel. Il faut établir que Voltaire et son hypothétique 'modèle' se sont rencontrés. Il se trouve que, grâce à une très précieuse contribution de Curd Ochwadt ${ }^{3}$, nous savons que la présence de Meister dans ce lieu fondateur que fut Bückeburg, devenu dans le roman Thunder-ten-Tronkh, lors des deux séjours qu'y fit Voltaire, est parfaitement attestée. Ce qui était totalement inattendu, c'est que Meister a enregistré sur le champ dans ses Mémoires ${ }^{4}$ non seulement ses jugements sur Voltaire, mais un compte rendu détaillé, quasi sténographique, de leurs entretiens de la journée. Or, la teneur de ces conversations atteste que des relations directes et conflictuelles se sont instaurées entre ces deux personnalités, tout spécialement à propos de problèmes tels que ceux des rapports de la morale et de la religion, de l'origine du mal et de la Providence.

(1) Dans un article paru dans la Revue d'Histoire Littéraire de la France, 98-1 (1998), p. 63-83, sous le titre «Aux origines de Candide: Une "économie de roman'». Les documents utilisés pour étayer l'hypothèse consistent essentiellement dans la correspondance complète de Voltaire et de la comtesse Bentinck; cf. n. 29

(2) Voir F. Deloffre, De la comtesse d'Empire à la baronette: Mme Bentinck et Cunégonde, dans les Mélanges offerts au professeur Jean-Paul de Nola, à paraître en 2004.

(3) Voltaire und die Grefen zu Schaumburg-Lippe (Jacobi Verlag, Bremen und Wolfenbüttel, 1977).

(4) Conservés sous le nom de Tagebücher Jobann-Heinrich Meisters à la Zentralbibliothek de Zurich, Ms. J 475. 
Il est donc légitime de procéder à une comparaison entre la figure livresque de mâ̂tre Pangloss et la personne réelle du théologien Meister. Certains estimeront qu'un parallèle même poussé ne peut constituer une 'preuve' (laquelle est de toute façon pratiquement hors de portée en ces matières $\left.{ }^{5}\right)$ : du moins donnera-t-il une idée du genre de personnage que Voltaire pouvait avoir à l'esprit en imaginant Pangloss. Ce qui n'est pas sans intérêt si on se souvient que les prétentions «réalistes» de l'auteur dans ce roman ont été souvent soulignées.

Le premier terme de la comparaison envisagé sera le personnage du roman, Pangloss. Le nom sous lequel il se présente peut être interprété de plusieurs manières: il ne répond pas en effet à un modèle de mots composés 'classique'. Disons qu'il suggère un personnage qui exerce sa langue sur tout sujet, qui trouve moyen de donner réponse à tout; ce trait, le plus caractéristique du personnage, est illustré par de nombreux passages, dès le premier chapitre du roman.

Après le nom vient la fonction. Elle apparaît dans ce passage:

Le fils du baron paraissait en tout digne de son père. Le précepteur Pangloss était l'oracle de la maison, et le petit Candide écoutait ses leçons avec toute la bonne foi de son âge et de son caractère (ch. I).

Le nom de Pangloss apparait juste après la mention du fils du baron. Comme Candide n'est dans la maison qu'en tant que neveu bâtard du baron, ce qui rend douteux qu'un précepteur lui soit attaché, mais qu'il est dit pourtant au chapitre VIII, p. 45, qu'il a été «élevé par le docteur Pangloss», on peut supposer que ce dernier a présidé généralement à l'éducation des enfants de la maison comme «haut précepteur».

Il a été question plus haut du «grand aumônier du baron», lequel est aussi le «vicaire du village». Faut-il entendre que le précepteur Pangloss se confond avec cet aumônier? Voltaire s'est gardé de le préciser: le faire aurait inutilement souligné le caractère antichrétien des attaques contre Pangloss. Mais l'hypothèse est appuyée par l'énoncé de la matière enseignée par le précepteur, la fameuse 'métaphysico-theologo-cosmolonigologie'.

Si, en pays protestant, 'l'oracle de la maison' professe des thèses théologiques, c'est qu'il est, sinon prêtre, du moins ecclésiastique, et que son enseignement prend la voie de la prédication.

Du point de vue intellectuel et moral, il est entendu que Pangloss est un fantoche. Philosophe et docteur comme ceux de Molière, il raisonne sans tenir compte des faits et persiste dans ses opinions même quand elles ont été démenties. Mais le caractère burlesque de ses discours ne le prive pas totalement des marques d'un homme réel. Si Voltaire ne fait pas de lui un portrait en pied, il lui prête dans le cours du récit certains attributs, un bon cœur, un certain bon sens, des capacités, des ambitions. On apprend ainsi que Pangloss à Constantinople est «au désespoir de ne pas briller dans quelque université d'Allemagne» ${ }^{6}$. Intellectuellement, il «écrit bien», «sait parfaitement l'arithmétique» ${ }^{7}$. Son érudition en matière d'histoire sacrée et profane, qui brille lorsqu'il fait le tableau des misères causées par les grandeurs humaines ${ }^{8}$, ne l'empêche pas de juger sainement des problèmes militaires contemporains'. Dans la vie courante, il ne manque pas d'esprit: Candide regrette son absence

(5) Ce problème a été abordé dans un article de F. DELOFFre, Quelques réflexions sur la critique d'attribution, L'Histoire littéraire: ses méthodes et ses résultats, Mélanges offerts à Madaleine Bertaud (Genève, Droz, 2001), notamment pp. 247-248.

(6) Ch. XXX, p. 150. Les références sont à l'edition Gallimard, Folio classique (2003).
(7) Ch. IV, p. 33.

(8) Ch. XXX, p. 154.

(9) «On peut assurer que quand trente mille hommes combattent en bataille rangée, il y a environ vingt mille vérolés de chaque côté», explique-til à Candide (ch. IV, p. 33). 
quand il doit prendre des mesures d'urgence ${ }^{10}$, et Pangloss trouve une solution de compromis lorsqu'il s'agit d'épouser Cunégonde en dépit d'un déficit en quartiers de noblesse ${ }^{11}$. Moralement enfin, il est vrai que le philosophe est présenté comme libidineux, mais c'est un rôle que la tradition lui attribue à l'époque: sans parler du Pédant joué de Cyrano de Bergerac, la «raison suffisante» du docteur Pangloss a son pendant dans le «syllogisme» du pédant Hortensius de Marivaux dans la seconde Surprise de l'amour. Du reste, ce trait n'empêche pas Pangloss d'aimer son élève et de veiller sur lui: l'affection que lui porte en retour Candide est certainement justifiée. Rien en un mot ne dément en lui la devise que lui prête son élève, à savoir que «les hommes ne sont faits que pour se secourir les uns les autres ${ }^{12}$. C'est dans la mesure où Pangloss n'est donc pas qu'une caricature qu'il est intéressant de le rapprocher du personnage réel de Meister, connu, on l'a dit, par les travaux de Curd Ochwadt ${ }^{13}$. Johan-Heinrich Meister, qui se fit appeler Henri Le Maître, était né vers 1700 aux environs de Zurich, fut d'abord prédicateur de la colonie française de Bayreuth. Il vint à Bückeburg en 1733. Il y avait été appelé par le comte régnant Albrecht-Wolfgang: dès ses années d'études à Genève, il avait commencé à prendre part à l'éducation des enfants de celui-ci en formant avec lui le projet d'un programme d'études. A Bückeburg, où il était chapelain et prédicateur (Hofprediger), il devait succéder à Beschefer, resté en Hollande, comme 'haut-précepteur' (Hofmeister) de Georges et de Wilhelm, fils du comte. Après la mort de l'aîné Georg (1742), il resta en bons termes avec Wilhelm. Une lettre de celui-ci à Mme Bentinck du 21 mai $1755^{14}$ montre que Meister lui avait offert un exemplaire de son Traité de religion ou Religionstraktat:

Pour ce qui est de M. Le Maître, il peut hardiment dire qu'il m'a éduqué. Je crains seulement que cela ne recommande pas son traité. Pour moi je me trouve honoré de son souvenir. Faites-lui bien mes compliments. Je souhaite que son ouvrage convertisse les incrédules.

La comtesse avait alors quitté Berlin et se trouvait à Leipzig où elle était l'amie du célèbre Gottsched et de sa femme. Gottsched était depuis 1730 professeur de poésie à l'université, et depuis 1734 professeur de logique et de métaphysique. Si, comme Pangloss, Meister qui était alors pasteur à Küssnacht, aux environs de Zurich, rêvait d'enseigner dans une université allemande, une démarche auprès de Gottsched, avec la recommandation de la comtesse Bentinck et du comte de Schaumburg-Lippe était un moyen tout indiqué d'explorer les voies pour y parvenir.

Quelques remarques permettent de comprendre comment un personnage nullement méprisable tel que Meister peut devenir, sous la plume de Voltaire, un Pangloss. La première raison est extérieure et tient, tout naturellement, au propos satirique du romancier. Ce propos l'incite à dégrader systématiquement le monde qu'il met en scène. L'exemple le plus frappant en est l'emploi de baron, avec les désignations annexes de baronnie, baronnette, etc. Dans une province comme la Westphalie, qui compte plusieurs principautés d'Empire, «le plus grand baron de la province» est manifestement au moins un comte, non un baron. De même, dans le comté de Schaumburg-Lippe, le chapelain et prédicateur de la cour Meister, qui prend le thé avec les seigneurs du château, n'est apparemment pas «vicaire du village». Il était en fait le chef de la communauté protestante de la petite ville de Bückeburg. On a vu aussi que

(10) Ch. IX, p. 48.

(11) Ch. XXX, p. 149.

(12) Ch. II, p. 24.

(13) Outre le petit livre signalé à la note 3 , nous avons aussi recouru au magistral ouvrage du même auteur, Wilhelm Graf zu Schaumburg-Lippe, Schrif- ten und Briefe, Veröffentlichungen der Leibiz-Archive (Viktorio Klostermann, Frankfurt am Main, 1977), 3 vol. in-8.

(14) Publiée par Curd Ochwadt dans l'ouvrage signalé à la note précédente, t. III (Briefe), pp. 90-91. 
la tradition littéraire expliquait les mœurs libidineuses attribuées à Pangloss, alors que Meister était marié et père de deux filles. Un autre facteur, spécifique cette fois, a peut-être aussi joué. Dans la petite cour de Bückeburg, Meister semble avoir passé, auprès de la «jeune génération», celle de Mme Bentinck et des fils du comte, pour un personnage quelque peu ridicule, une «tête de Turc» en quelque sorte. C'est ce qui ressort d'une composition que Mme Bentinck, son auteur, fit malicieusement circuler sous le nom de Meister. Il s'agissait d'un pastiche de l'Epître à Uranie de Voltaire. Il était déjà impertinent d'attribuer à Meister une part dans cette œuvre fort peu chrétienne. Il l'était encore plus de lui attribuer la paternité de ce pastiche franchement grossier ${ }^{15}$. Composé en 1745, il ne fut pas connu de Voltaire à ce moment; mais Mme Bentinck ou Wilhelm Schaumurg-Lippe peuvent lui en avoir parlé pendant le séjour de Berlin. De toute façon, la satisfaction que Meister éprouvait de sa personne - on en jugera plus loin - l'exposait manifestement à l'ironie de Voltaire.

Du reste, sur le plan du statut social, les ressemblances entre roman et réalité sont frappantes. Le journal de Meister montre bien à quel point il est, comme Pangloss, «l'oracle du château». Ainsi, lorsque, juste avant la première visite de Voltaire, le comte Wolfgang de Schaumburg-Lippe peut se procurer deux versions de la Critique du Prince de Machiavel, il invite le chapelain, «par grâce spéciale», deux jours de suite (14 et 15 novembre 1740) à venir en écouter la lecture qu'il en fait avec Mme Bentinck et à formuler ses opinions sur l'ouvrage de Frédéric. Elles sont résumées chaque fois dans le Journal de Meister en une ligne, le premier jour:

Charmé de l'esprit de l'auteur. Déploré son dégoût pour la révélation ${ }^{16}$

et le second:

Trouvé encore des preuves de l'esprit brillant mais perverti de l'auteur ${ }^{17}$.

Meister est si content de ses observations qu'il en fait un précis ${ }^{18}$ qu'il lit en dehors de la petite cour à d'autres compagnies de Bückeburg. Pourtant, lors de la première visite de Voltaire, du 8 au 10 décembre 1740, même lorsqu'il y fut présent, il ne prit qu'une part minime aux entretiens. Comme le remarque Curd Ochwadt ${ }^{19}$, les principaux interlocuteurs de Voltaire, le comte régnant Wolfgang et Mme Bentinck, sa favorite, étaient, par leur culture, leur ouverture d'esprit et leur personnalité, de nature à ne pas avoir besoin de lui pour soutenir la conversation. Mais cela n'empêcha pas Meister de noter ses impressions, et elles sont instructives. Dès le 8 décembre, jour de l'arrivée du voyageur, il écrit:

Allé au château. Conversation avec M. de La Croisette [le gouverneur militaire du château] et avec les dames. Affaires. Vu là M. de Voltaire en passant, comme à son retour de Berlin il s'était détourné exprès pour faire sa cour ici. Compliments suspects. Recommandé inutilement par S.E. [Albert Wolfgang] qui me parla des discours du poète et de la comédie qu'il avait faite nouvellement de Mahomet.

Quoique on puisse hésiter sur la personne qui a été «recommandée inutilement» par le comte, Voltaire à Meister ou l'inverse, il est clair que les «compliments sus-

(15) Il est reproduit intégralement avec un commentaire par C. OCHWADT, Voltaire un die Grafen zu Schaumburg-Lippe, pp. 55-64.

(16) C. OCHWADT, op. cit., p. 27.

(17) Ibid., ibid.
(18) On le connaît sous le titre de Lettre sur l'Antimachiavel, qu'il lira notamment à Mme Beschefer, femme de son prédécesseur au préceptorat des fils du comte; voir Ochwadt, op. cit., p. 27.

(19) Ibid., p. 29. 
pects» sont ceux que l'hôte du château a adressés ironiquement au chapelain. Du reste, lorsque Voltaire a quitté le château le dimanche 11 décembre 1740, le journal de Meister laisse clairement paraître son hostilité envers lui. Après avoir délivré son prêche «très heureusement en présence de la cour», le prédicateur est invité à dîner par le comte Frédéric, frère cadet d'Albert Wolfgang:

Conversation avec M. le comte Frédéric des discours de Voltaire (...) et remis au même mon sermon. Dîné avec S.E. etc. (...) Enfin visite à Mme la comtesse douairière. Remis la relation de l'état de ma femme. Conversation des discours impies de Voltaire, item de mon sermon ${ }^{20}$.

On peut penser que Meister prit le temps de réfléchir à la visite de Voltaire. Se sentant peu capable de rivaliser avec lui dans le domaine du brillant, il se rabattit sur le solide. En terre réformée, la religion était le meilleur fonds. Un auto-portrait qu'il composa en 1742 pour l'envoyer à la princesse de Nassau-Siegen comportait une sorte d'apologie dont chaque terme est aussi une pointe contre l'esprit voltairien:

J'attribue aux progrès modiques que Dieu m'a fait faire dans mon christianisme le peux de cas que je fais de la vivacité, de la fécondité et de la beauté d'imagination qui fait ce qu'on appelle les beaux génies et les beaux esprits, quoiqu'on m'ait cajolé là-dessus d'une manière qui exposait mon amour-propre à de grandes tentations. Bien loin de cultiver cette souplesse d'esprit pour briller par là dans le monde, je ne me permets de faire valoir ce que je peux posséder de ce talent qu'autant que cela peut servir au solide en montrant qu'il n'y a rien de plus beau, ni même de véritablement beau, que ce qui est bon ${ }^{21}$.

C'est armé de ces réflexions salutaires que Meister aborda la seconde rencontre avec Voltaire où il allait cette fois tenir une place de choix. Pour cette visite, en octobre 1743, le comte régnant et Mme Bentinck étaient absents. La protectrice de Meister, la pieuse princesse Charlotte-Amélie de Nassau-Siegen, femme délaissée du comte, l'invita à prendre part aux entretiens. Il y tint sans conteste la vedette devant les hôtes du château. Voltaire était arrivé à Buckeburg de bonne humeur ${ }^{22}$ le dimanche 20 octobre dans l'après-midi. Dès le soir du même jour, Meister jette à la hâte dans ses carnets une brève notice qui va lui servir d'aide-mémoire les jours suivants pour développer et mettre au net la teneur des entretiens:

visite avec ma famille à $\mathrm{M}$. Cleve. Bu là le café (...) Conversation de nouvelles (...) Ouï que M. de Voltaire était arrivé au château. Quitté à cette occasion la compagnie.... Enfin allé au château. Raisonné, en attendant M. de Voltaire, avec M. de La Croisette. Puis lié conversation avec M. de Voltaire après qu'il eut écrit à $M$. de Podewils et Mme la marquise du Châtelet. Raisonné de la cour de Bayreuth, de celle de Brunswic, de la bibliothèque de Wolfenbüttel, de celle du Vatican, de celles de Paris. Puis continué dans la chambre de S.A.S. ${ }^{23}$ à raisonner du Poème de Racine, de Pamela, des Anglais, de l'esprit sectaire, du père La Canaye, de l'esprit de persécution, de la perpétuité et divinité de la morale, des païens, des martyrs accusés de cabales, des Huguenots. Soupé avec S.A.S. et M. de Voltaire (...) Conversation de la persécution de France, du czar de Russie, des porteurs de deux écharpes ${ }^{24}$, de la paix etc. Après souper con-

(20) Ibid., p. 28.

(21) Ibid., p. 57.

(22) Dans une lettre datée «de Buckeburg la Lippe ce 19[20?] [octobre 1743] au soir», il écrit à Podewils «Je voyage au rebours de Don Quichotte qui prenait toutes les hôtelleries pour des châteaux. Moi je prends tous les châteaux pour mes hôtelleries» (Besterman, D2869).

(23) La seconde femme du comte Wolfgang, née princesse de Nassau-Siegen.
(24) Allusion à deux histoires qu'a contées Voltaire, l'une d'un curé du canton de Glaros qui offre un prêche à ses paroissiens réformateurs et une messe à ceux qui souhaitent la garder, l'autre d'un anachorète d'une île de l'Archipel qui déploie une croix et un rosaire quand il voit arriver un vaisseau français ou espagnol, et qui à l'approche d'un vaisseau turc orne sa cellule d'un croissant en criant: «La en la illala ve Mouhammed oul resoul oulla» (sic) (OcHWADT, ibid., p.46). 
versation avec M. de Voltaire de ses ouvrages, de l'Antimachiavel, considération de l'impiété du roi etc. Conversation avec S.A.S. ${ }^{25}$

Ce résumé donne déjà une idée de la variété des sujets abordés et des compétences diverses de notre «maittre Pangloss». Mais Meister fait mieux. Le jour suivant, 21 octobre, il note soigneusement comment, en s'y reprenant à quatre reprises, il a recueilli et mis par écrit «les sujets de la conversation d'hier»: à savoir de la première fois, en prenant le thé «dans le poële du ménage en fumant une pipe», à la dernière après souper, une fois faite «la prière en famille», dans une «tabagie solitaire». Le fruit de ces travaux, intitulé Ma conversation avec Voltaire, est lu par Meister à sa femme le 22 octobre, puis commenté en présence de diverses personnes, dont la première, le 23 octobre, est la princesse Charlotte-Amélie:

Audience gracieuse de S.A.S. (...) Tristes réflexions. Conversation de M. de Voltaire, et de l'abus des talents de l'esprit ${ }^{26}$.

Le ton est donné. Ce n'est pas seulement la qualité tout à fait remarquable de la restitution par Meister de la conversation de Voltaire qui frappe ${ }^{27}$, c'est la façon, justement notée par Curd Ochwadt ${ }^{28}$, dont il tire à soi les entretiens et les dirige: qu'il soit en scène à la première personne ou s'abrite sous le voile d'un 'on', c'est bien lui qu'il met alors en scène: si à l' occasion un des membres de la petite société intervient, il est en effet cité nommément et avec ses titres.

Il est malheureusement impossible de reproduire ici la totalité de la «conversation» telle que l'a publiée Curd Ochwadt ${ }^{29}$. On y trouve une foule de thèmes voltairiens dont plusieurs réapparaîtront dans le Dictionnaire philosophique, notamment celui de la défense du paganisme et de la supériorité de la morale antique sur la morale chrétienne. On ne retiendra d'abord ici que ce qui, curieusement, revient dans Candide. C'est par exemple, après l'évocation du carnaval de Venise, celle d'un «cordelier gaillard et bien nourri» qui «entretien[t] une catin en cachette et peut-être plusieurs» ${ }^{30}$; d'un «maudit autodafé» auquel le roi de Portugal assiste et «où il a fait griller quinze pauvres misérables» ${ }^{31}$; d'une «femme sauvage qui venait d'un de ces pals de l'Amérique où l'on mange la chair humaine» et qui «la trouve «délicieuse» ${ }^{32}$; du czar Pierre premier qui exécuta de sa propre main «au-delà de mille sept cents conspirateurs» ${ }^{33}$; enfin, la comparaison du destin du «jeune ex-empereur Ivan» avec celui «de plusieurs princes et princesses de la maison Stuart» ${ }^{34}$, qui annonce de loin la rencontre des six rois à Venise, au chapitre XXVI du roman. Les points de la discussion qui révèlent le mieux les positions de Voltaire et de son contradicteur touchent à la religion. Voltaire se dit catholique: «car avec toute la liberté avec laquelle il se

(25) Ibid., p. 57.

(26) Ibid., p. 34

(27) Voir l'excellent commentaire de Curd Ochwadt sur ce point, ibid., p. 35.

(28) Cf. Id., ibid., «Den Aufzeichnungen zufolge hat Meister das Gespräch weitgehend an sich gezogn und geführt».

(29) Ibid., pp. 36-49. On en trouvera de larges extraits dans Voltaire et sa grande amie. Correspondance complète de Voltaire et de Mme Bentinck (paru à la Fondation Voltaire, Oxford, 2003), Prologue, «Un château en Westphalie».

(30) Ibid., p. 40; comparer Candide, ch. XXIV, pp. 121-126, la rencontre avec le théatin «frais, potelé et vigoureux» et de Pâquette devenue elle aussi une «catin».

(31) Ibid. p. 42; comparer Candide, ch. VI.

(32) Ibid., ibid., comparer au chapitre XVI (les Oreillons) de Candide les réflexions de Cacambo, «il vaut mieux manger ses ennemis que d'abandonner aux corbeaux et aux corneilles le fruit de sa victoire» (p. 77).

(33) Ibid, p. 45; comparer Candide, ch. XII: «ce seigneur ayant été roué au bout de deux ans avec une trentaine de boyards pour quelque tracasserie de cour...».

(34) Ibid., p. 47. 
raisonne sur la religion, il se donne pour bon catholique romain ${ }^{35}$. Les deux hommes s'accordent à peu près pour reoonnaître que si le «fanatisme» caractérise le catholicisme, l'esprit de secte appartient en propre au protestantisme. Si l'on se demandait la raison de l'épisode où, au chapitre III du roman, un 'orateur' protestant maltraite Candide parce qu'il doute que le pape soit l'Antéchrist, la réponse est que Voltaire veut dans une certaine mesure se distinguer des réformés, qui cherchent parfois à l'annexer.

Le chapitre le plus notable de la Conversation concerne pourtant la théosophie, qui deviendra, pour Voltaire, la 'métaphysico-theologo-cosmolonigologie'. On y entend, mieux que nulle part ailleurs, comme la voix de Voltaire. Significativement, il a été introduit par Meister:

Après souper, on s'arrêta encore un peu dans l'antichambre de S.A.S. M. de Voltaire avait une lettre à expédier à $\mathrm{M}$. de Bessel, directeur de la chambre des domaines à Minden, pour commander les chevaux de corvée que le roi donnait à notre voyageur dans tous ses états. On lui dit que Madame de Bessel était fille d'un officier français réfugié, M. de Béquignoles, mort à Magdebourg. Ce discours me donna l'occasion de parler des ouvrages que cet officier avait écrit pour expliquer l'origine et la permission du mal par le mystère de la Sainte Trinité, selon les principes de Jacob Böhme. M. de Voltaire ne savait rien de ce Böhme. Comme je lui dis que ç'avait été un cordonnier allemand, auteur de plusieurs ouvrages mystiques et philosophiques, ou, comme il pourrait les appeler fanatiques. - Cordonnier mystique! répliqua M. de Voltaire d'un ton railleur, voilà qui est merveilleux. Il était sans doute inspiré. Et pourquoi les cordonniers allemands ne le seraient-ils pas aussi bien que les pêcheurs de Galilée? Ah! Monsieur, lui répondis-je, quand les cordonniers allemands prouveront leur inspiration par des raisons aussi fortes que le sont celles qui constatent l'inspiration des pêcheurs galiléens, nous les écouterons. Mais vous me permettrez de vous dire que l'inspiration divine n'est pas une matière de raillerie ${ }^{36}$.

Certes, Jakob Böhme n'est pas l'inventeur de l'optimisme, mais ses recherches visaient à rendre compte du problème du mal en réunissant le bien et le mal, comme le oui et le non, l'ombre et la lumière, dans une unité spirituelle originelle. Ses spéculations portaient aussi sur le problème de la Sainte Trinité: comment un dieu triple était émergé d'un pouvoir unique primitif. Comme le système de l'optimisme leibnizien, qui en procède d'une certaine façon, la doctrine de Böhme admet une providence. C'est du reste l'ensemble de la pensée théosophique que vise Voltaire lorsque, par exemple, il prête cette réflexions à Candide:

C'est bien dommage, disait Candide, que le sage Pangloss ait été pendu contre la coutume dans un autodafé; il nous dirait des choses admirables sur le mal physique et sur le mal moral qui couvrent la terre et la mer, et je me sentirais assez de force pour lui faire respectueusement quelques objections ${ }^{37}$.

Mais un tel sujet serait tout à fait impropre à figurer dans un roman. Si Voltaire retient l'optimisme pour objet quasi unique de ses attaques, c'est qu'aucune discussion n'est ici nécessaire. Les faits se chargent de la démonstration de la façon la plus comique et la plus irréfutable.

Pour en revenir à Jean-Henri Le Maître, ou Heinrich Meister, ce qui nous intéresse consiste moins à déterminer ce qui dans sa pensée a pu hérisser Voltaire qu'à reconnaître ce qui a pu subsister de son personnage dans la figure du héros de roman. À en juger par ce dernier, Voltaire a pu éprouver pour le chapelain de Bückeburg une certaine indulgence, non exempte d'ironie et de quelque mépris. Quant à Meister, 
c'est en portant sous son récit de la Conversation avec Voltaire un bref poème épigrammatique, dont il ne donne pas la source, qu'il fait ses adieux à Voltaire:

Vers d'un auteur anonyme sur le voyage de M. de Voltaire à Berlin

Un nourrisson de Melpomène,

Dont la religion, la cour, ni le bon sens,

N'ont jamais pu tenir [tarir ?] l'impétuose [sic] veine,

Ira rimer aux pays protestants.

Ce prompt départ fâchera bien des gens.

Mais c'est à tort; car ce n'est qu'une astuce

Fort ordinaire aux beaux esprits:

C'est que craignant encor qu'on le brûle à Paris,

L'auteur aimera mieux s'aller morfondre en Prusse ${ }^{38}$.

Sans y penser, maître Meister ouvrait ainsi à Voltaire, au sortir de Thunderten-Tronkh, une perspective sur les aventures de son héros au second chapitre de Candide.

FRÉDERIC DELOFFRE

(38) Cité par C. Ochwadt, Voltaire und die Grafen zu Schaumburg-Lippe, p. 49. 